\title{
RESONANT CONDUCTANCE OF TWO-DIMENSIONAL ELECTRON SYSTEMS
}

\author{
A. Matulis and K. Patiejūnas \\ Semiconductor Physics Institute, Lithuanian Academy of Sciences \\ Goštauto 11, 2600 Vilnius, Lithuania
}

The ballistic conductance of two-dimensional ring calculation results are presented. The influence of the magnetic field and electron elastic scattering on the resonant ring conductance are discussed.

PACS numbers: 73.20.Dx, 72.20.Dp

The properties of the ballistic quantum two-dimensional (2D) electron systems are of intense current interest. After Wharam and van Wees discovered $[1,2]$ the phenomenon of the conductance quantization in a narrow channel of $2 \mathrm{D}$ electron gas of a GaAs- $\mathrm{Ga}_{x} \mathrm{Al}_{1-x} \mathrm{As}$ heterojunction, a great number of various phenomena related to the interference of electron waves in 2D channels and resonant electron tunnelling have been discovered. We propose [3] a special method for describing the above-mentioned phenomena and calculating the device conductance, based on the Kirchhoff integral technique analogous to that used in the diffraction theory [4].

In this paper the results of the 2D ring quantum ballistic conductance calculation based on the application of that Kirchhoff integral technique and its generalization in the case of homogeneous magnetic field are presented. This method is of advantage when the 2D device consists of some parts interconnected by small apertures as shown in the inset of Fig. 1. The ballistic conductance of such a device can be calculated by means of the electron wave functions $\Phi^{L, R}(x)$ defined at the apertures $L, R$. In the long-wave approximation, when the electron wavelength $\lambda=2 \pi / k=2 \pi / \sqrt{2 m \varepsilon_{\mathbf{F}}}\left(\varepsilon_{\mathrm{F}}\right.$ is the electron Fermi energy) is much greater than the aperture dimensions $d$, these aperture functions are of the following form:

$$
\Phi^{L, R}(x) \propto \Phi^{L, R} \sqrt{1-(2 x / d)^{2}},
$$

where the aperture amplitudes $\Phi^{L, R}$ satisfy the equation set:

$$
\begin{aligned}
& \left(1-\mathrm{i} \eta+K^{L, L}\right) \Phi^{L}+K^{L, R} \Phi^{R}=1, \\
& K^{R, L} \Phi^{L}+\left(1-\mathrm{i} \eta+K^{R, R}\right) \Phi^{R}=0 .
\end{aligned}
$$

Here

$$
\eta=(\pi / 8)(k d / 2)^{2}
$$

and

$$
K^{A, B}=\left.(\pi / 4)(d / 2)^{2}\left[\partial^{2} /\left(\partial z \partial z^{\prime}\right)\right] G\left(r \mid r^{\prime}\right)\right|_{x=x^{\prime}=0, z=z_{A}, z=z_{B}} .
$$




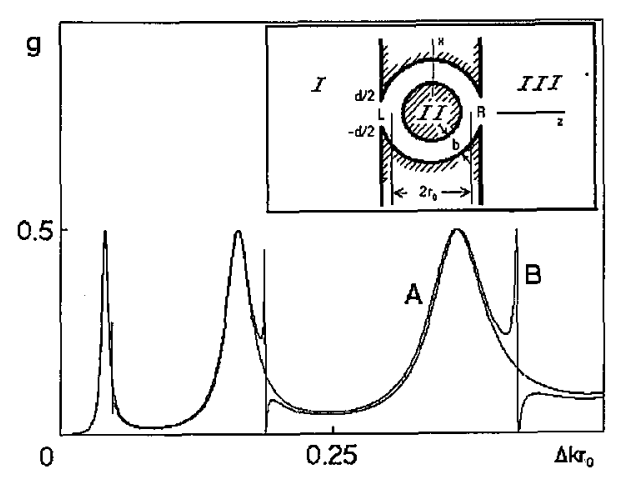

Fig. 1. The first radial mode contribution to the ring conductance as a function of $\Delta k r_{0}=\left(k-k_{1}\right) r_{0}$ for $b / r_{0}=0.3$ and $d / b=0.8$; curve $A, \xi=0$; curve $B, \xi=0.1$. The layout of the $2 \mathrm{D}$ ring in the inset.

Symbol $G\left(r \mid r^{\prime}\right)$ stands for the Green function of the device central part II calculated with the closed apertures, and $z_{A}$ and $z_{B}$ are the coordinates of the appropriate apertures.

If $V$ is the voltage applied to the half planes I and III and $J$ is the current through the device, the dimensionless conductance can be represented as follows:

$$
g=\left(\pi \hbar / e^{2}\right) J / V=g_{0}\left|\Phi^{R}\right|^{2},
$$

where $g_{0}$ is the conductance of the device consisting of a single aperture.

Solving Eqs. (2) and substituting for expression (5) we write the following final expression for the device conductance:

$$
g=g_{0}\left|\frac{K^{R, L}}{\left(1-\mathrm{i} \eta+K^{L, L}\right)\left(1-\mathrm{i} \eta+K^{R, R}\right)-K^{R, L} K^{L, R}}\right|^{2} .
$$

Making use of that expression the ring conductance in two different cases is considered. The first one is the case with the magnetic flux $\Phi$ penetrating the ring and the second one is the case when the entire device is situated in the homogeneous magnetic field $\boldsymbol{H}$. For the sake of simplicity the narrow ring case with $d \ll b \ll r_{0}$ is considered.

In the first case with the magnetic flux $\Phi$ penetrating the ring the Green function equation with the Hamiltonian

$$
\widehat{H}=-(1 / 2)\left[\partial^{2} / \partial r^{2}+\left(1 / r_{0}^{2}\right)(\partial / \partial \varphi-\mathrm{i} \xi)^{2}\right],
$$

is solved, where $\xi=e \Phi / 2 \pi \hbar c$ is the dimensionless magnetic flux. In that case $g_{0}=\eta^{2}$ and the solution of the Green function equation lead to the following expressions for the coefficients: 


$$
\begin{aligned}
& K^{L, L}=K^{R, R}=\sum_{n=1}^{\infty} A_{n}(k) \frac{\sin \left[\pi \lambda_{n}(k)\right] \cos \left[\pi \lambda_{n}(k)\right]}{\sin \left(\pi \lambda_{n}^{+}\right) \sin \left(\pi \lambda_{n}^{-}\right)}, \\
& K^{L, R}=K^{R, L}=-\sum_{n=1}^{\infty} A_{n}(k) \frac{\sin \left[\pi \lambda_{n}(k)\right] \cos (\pi \xi)}{\sin \left(\pi \lambda_{n}^{+}\right) \sin \left(\pi \lambda_{n}^{-}\right)}
\end{aligned}
$$

where

$$
\begin{aligned}
& A_{n}(k)=\left[\pi r_{0} / 4 b \lambda_{n}(k)\right]\left(k_{n} d / 2\right), \\
& \lambda_{n}(k)=r_{0} \sqrt{k^{2}-k_{n}^{2}}, \quad \lambda_{n}^{ \pm}=-\xi \pm \lambda_{n}(k), \quad k_{n}=\pi n / b .
\end{aligned}
$$

Coefficients (8) and consequently the ring conductance (6) consist of series of spikes corresponding to each ring radial mode. In Fig. 1 the first radial mode $(n=1)$ contribution to the ring conductance as a function of $\Delta k r_{0}=\left(k-k_{1}\right) r_{0}$ is presented. With the magnetic flux absent (curve A) each eigenvalue (10) is doubly degenerate, what corresponds to two equivalent electron motions in the ring: clockwise and counterclockwise. When the magnetic field is switched on, these electron motions are no longer equivalent, the eigenvalue degeneracy disappears and all spikes become split (curve B). It is worthwhile to note the very specific kind of spikes splitting: the spike wings rather than the tops are split.

The proposed method enabled us to take into account the elastic electron scattering in the ring [5]. The elastic scattering influence on the resonant ring conductance was considered by means of including the additional impurity potential into Hamiltonian (7). In the single radial mode approximation this leads to the coefficient expressions similar to (8). The elastic electron scattering was shown to cause the same type spike splitting as the magnetic field. It is quite natural, since the presence of an impurity removes the above-mentioned eigenvalue degeneracy. There are, however the specific impurity locations when the conditions $\sin \left(m \varphi_{0}\right)=0$ or $\sin \left(m \varphi_{0}\right)= \pm 1$ ( $\varphi_{0}$ being the angle between the aperture and the impurity location and $m$ is the spike number) are satisfied. In that case the conductance spike corresponding to the $m$-th angular mode is not split. For illustration the second spike splitting caused by the elastic scattering is shown in Fig. 2. The curve A shows the conductivity in the case without magnetic flux through the ring. When the magnetic field is switched on, the spike splitting increases. When the magnetic field becomes large enough, the interaction between the neighbouring spikes appears what leads to a rather complicated spike shape (curve B) resembling the chaotic oscillations commonly present in Aharonov-Bohm effect in metallic ring with numerous impurities.

In the second case when the entire device (the ring II and half-planed electrodes I and III), is situated in a homogeneous magnetic field $\boldsymbol{H}$, the Hamiltonian

$$
\widehat{H}=-(1 / 2)(\nabla-\mathrm{i} e \boldsymbol{A} / c \hbar)^{2},
$$

where

$$
\boldsymbol{H}=\operatorname{rot} \boldsymbol{A} .
$$




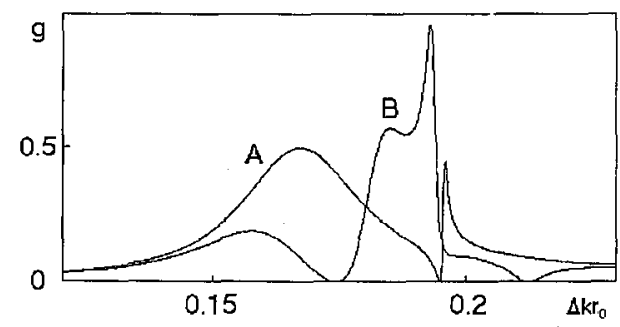

Fig. 2. The second spike splitting by the elastic electron scattering $\left(b / r_{0}=0.3 ; d / b=\right.$ 0.8 ). The impurity situated at $\varphi_{0}=\pi / 3$, the effective scattering strength 0.5 . Curve $A$, $\xi=0 ;$ curve $B, \xi=0.1$.

In this case the single aperture conductance is contributed only by those half-plane edge eigenstates whose energy is higher than $\varepsilon_{\mathrm{F}}$. Thus, this conductance displays the following step-like energy dependence:

$$
g_{0}=\eta^{2}\left(4 / k^{4}\right) \sum_{n, n^{\prime}} \Theta\left(k^{2} / 2-n\right) \Theta\left(k^{2} / 2 n^{\prime}\right), \quad \Theta(x)= \begin{cases}1, & x \geq 0 \\ 0, & x<0\end{cases}
$$

It is qualitatively similar to the conductance of a saddle-point constriction in a magnetic field considered in [6].

The ring conductance essentially depends on the relation between the ring width $b$ and the magnetic length $\lambda_{H}=\sqrt{c \hbar / e H}$. When $b \ll \lambda_{I}$ the ring radial eigenfunctions have the similar bulk shape as in the previously considered case. Therefore, the ring conductance shows the similar resonant behaviour and similar spike splitting caused by the magnetic field. In the opposite case $b \gg \lambda_{H}$, only the electron edge eigenstates corresponding to the electron motion along the outer ring border contribute to the ring conductance. In this case the angular motion is no more degenerate and, thus, neither magnetic field nor the elastic impurity scattering splits the spikes. The coefficients $K^{A, B}$ now have the form (8) with $\lambda_{n}^{+} \equiv \lambda_{n}(k)$ and $k_{1}^{2} / 2$ replaced by corresponding radial mode eigenvalue. Therefore, the conductivity looks like that represented by curve A in Fig. 1.

\section{References}

[1] D.A. Wharam, T.J. Thornton, R. Newbury, M. Pepper, H. Ahmed, J.E.F. Frost, D.G. Hasko, D.C. Peacock, D.A. Ritchie, G.A.C. Jones, J. Phys. C, Solid State Phys. 21, L209 (1988).

[2] B.J. van Wees, H. van Houten, C.W.J. Beenaker, J.G. Williamson, L.P. Kouwenhoven, D. van der Marel, C.T. Foxon, Phys. Rev. Lett. 60, 848 (1988).

[3] A. Matulis, K. Patiejūnas, J. Phys., Condens. Matter 3, 553 (1991).

[4] H.A. Bethe, Phys. Rev. 66, 163 (1944).

[5] A. Matulis, K. Patiejūnas, Litovskii Fizicheskii Sbornik 31, 589 (1991).

[6] M. Búttiker, Phys. Rev. B 41, 7906 (1990). 